\title{
Bridge Performance in Asynchronous Cognitive Personal Area Networks
}

\author{
Md. Mizanur Rahman, Jelena Mišić and Vojislav B. Mišić \\ Ryerson University \\ Toronto, ON, Canada M5B 2K3 \\ Email: \{mdmizanur.rahman, jmisic, vmisic\}@ryerson.ca
}

\begin{abstract}
Bridging between two cognitive personal area networks (CPANs) is a fundamental step towards a reliable and efficient routing protocol. In this paper we describe a bridging protocol for two-hop cognitive networks that use the transmission tax-based MAC, and propose ways to prioritize bridge traffic over that of ordinary nodes in both CPANs. We develop an analytical model based on probabilistic modeling and queueing theory to evaluate the performance of the bridging protocol. We validate the network performance by analyzing the waiting time of local and non-local packets and how the node or bridge transmission is affected by the collision with primary source activity. Our results provide insight into the impact of various traffic and network parameters on the performance of two-hop bridging. The results indicate that the proposed protocol is effective and efficient when the bridge transfers moderate amount of traffic.
\end{abstract}

Index Terms-opportunistic spectrum access; wireless personal area networks; channel hopping cognitive networks; bridging in two-hop networks

\section{INTRODUCTION}

Opportunistic or cognitive spectrum access paradigm strives to improve the utilization of available spectrum between licensed primary users (PUs) and unlicensed secondary users (SUs) [1]. SUs are often grouped in small networks or piconets, sometimes referred to as Cognitive Personal Area Networks (CPANs) which use collaborative spectrum sensing [9] to guide adaptive channel hopping [6] that aims to minimize the interference from and to PUs.

Many theoretical and practical proposals focused on the creation, operation and performance of single hop networks. Recently, multi-hop networks have begun to attract research attention [7], [13]. Most of the effort was oriented towards routing, which is a well researched subject in ad hoc networks, but with two additional constraints. First, routes as well as ongoing data transmissions can be disrupted by collisions with primary user transmissions on the selected channels. Second, routes can be difficult to repair due to the lack of coordination among cognitive piconets that participate in the formation and maintenance of the route. Routing is frequently linked to the solutions of the spectrum decision (i.e., channel selection) and network coexistence problems, as witnessed by the joint routing and link scheduling approach in [14] or a sessionoriented spectrum trading system [13]. A distributed crosslayer optimization approach [16] is based on joint consideration of power control, scheduling, and routing. Recently, new routing metrics specifically tailored to cognitive networks have been proposed, including various flavors of spectrum temperature [5] and minimum-maintenance-cost routing [3], together with the associated routing protocols. While these results highlight many of the issues related to design, operation, and performance of routing protocols, the performance of multi-hop CPANs at the level of the underlying MAC protocols has not been adequately addressed.

In this paper, we consider a two-hop network that consists of two CPAN piconets, hereafter referred to as source and destination CPANs, or SCPAN and DCPAN, respectively. Both CPANs use the transmission tax-based MAC protocol in which nodes pay for packet transmission by performing spectrum sensing [10]. We assume that the two CPANs are aware of each other and that a shared node, hereafter referred to as the bridge, hops between SCPAN and DCPAN to deliver interCPAN traffic from the former to the latter. (We consider a single, unidirectional bridge because the traffic in the opposite direction can easily be accommodated by having another bridge node.) However, a multi-hop network can be formed when one or more CPANs are operating in between SCPAN and DCPAN and each pair of CPANs (from SCPAN to DCPAN) has a bridge node. Each bridge of different pair of CPANs is responsible to carry the data from SCPAN towards DCPAN. This can only be accomplished when a bridge is able to exchange data between two asynchronous CPANs since every CPAN operates independently and the starting time of each CPAN superframe might differ from other CPAN. In our previous work [15] we have considered a case of CPANs which operate synchronously so that their superframes start and finish at the same time; in this paper, we allow CPAN superframes to be skewed in time by an arbitrary interval. To ensure timely delivery of inter-CPAN traffic, bridge transmissions should be given higher priority in the DCPAN. This may be accomplished by scheduling these transmissions early in the superframe, as well as by servicing bridge traffic with a more efficient policy than the intra-CPAN traffic. However, both these techniques may degrade performance for ordinary nodes, and we investigate the performance of this arrangement with respect to both the inter- and intra-CPAN traffic, using probabilistic modeling and queueing theory.

The rest of the paper is structured as follows: Section II describes the operation of the CPAN and bridging between two CPANs. Section III presents the probabilistic model of bridging algorithm with transmission, sensing and reception by ordinary nodes. Access delay for both intra-CPAN and inter-CPAN traffic is discussed in Section IV. Results of the 


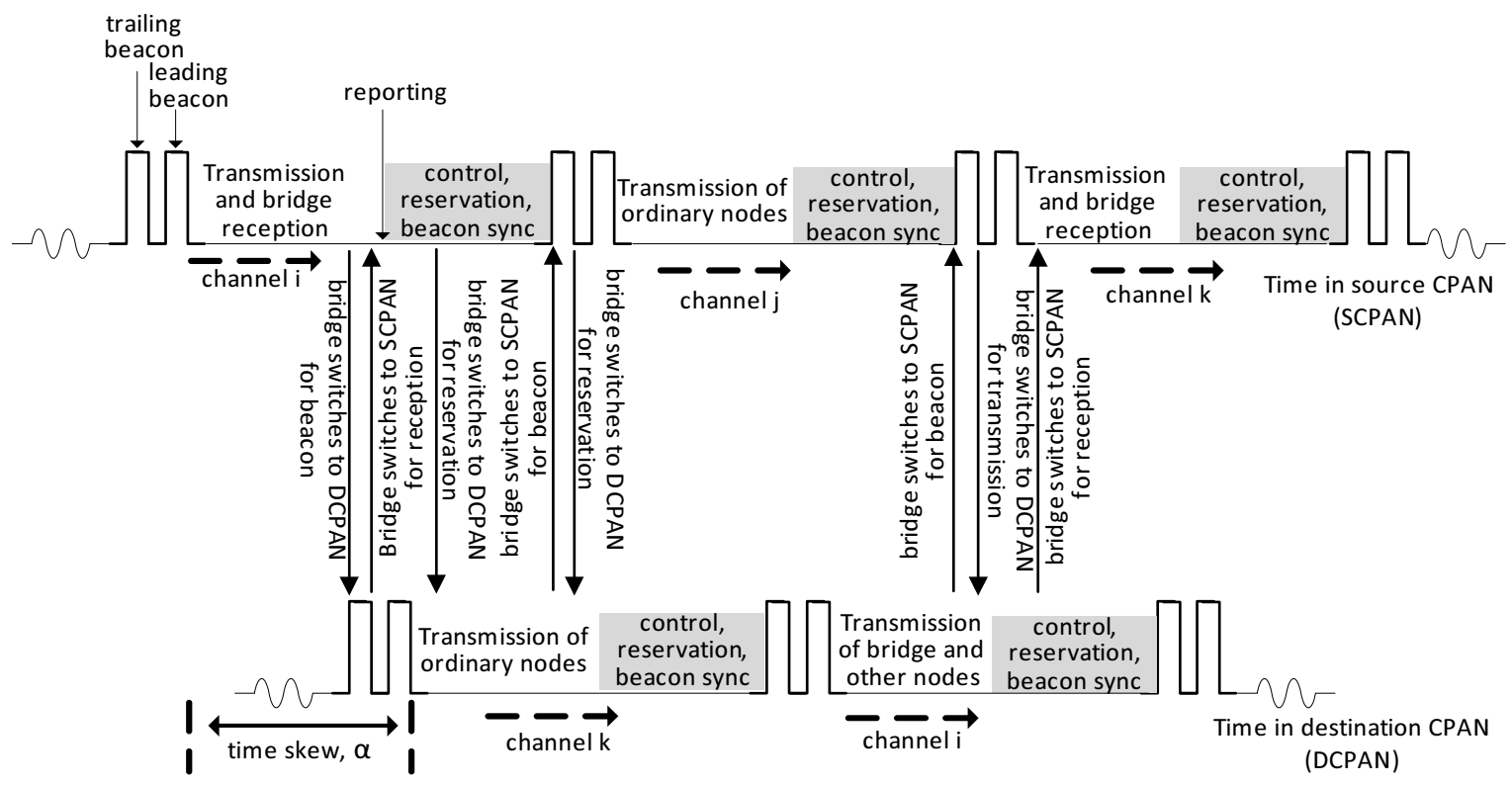

Fig. 1. Bridge operation.

performance analysis are presented and discussed in Section V. Section VI concludes the paper with stating future work.

\section{ON CPAN AND BRIDGE OPERATION}

Time in a CPAN that follows the transmission tax-based MAC protocol is divided into constant size superframes that begin and end with dedicated beacon frames, as shown in Fig. 1. Specific portions of the superframe are devoted to data transmission, reporting of sensing results, and sending bandwidth requests and other administrative activities.

Ordinary nodes request bandwidth from the coordinator which grants them access in a round-robin fashion and announces in the leading beacon which nodes will transmit and receive data, and which ones will perform sensing duty. Requests that can't be accommodated in the coming superframe will be deferred to subsequent ones.

Nodes that have been granted transmission slots pay by subsequently sensing some of the channels and sending the results to the coordinator. Sensing duration is determined as the product of the sensing penalty coefficient and the number of packets transmitted since the last sensing duty. A node can apply for bandwidth after it finishes the sensing duty and has data to transmit. When a node which is currently performing sensing duty has to receive data, it can suspend the sensing until it receives the data.

The coordinator uses sensing results to build and update the free channel table. using this table, the coordinator selects the working channel for the next-hop superframe [12] as well as a number of backup channels [11]. The next-hop and backup channels are announced in the trailing beacon. In this manner, all nodes in the CPAN hop together to the next channel, or attempt recovery in case of collision with a primary user transmission.

We assume that CPANs are aware of each other, as noted above. Since all nodes that had successfully transmitted a packet have to perform spectrum sensing, a node from one CPAN that detects another CPAN operating on a different channel can volunteer to become a bridge. The node needs to listen to that channel until it hears the trailing beacon which announces the next-hop and backup channels for the DCPAN. Once the bridge goes to the SCPAN in order to begin its operation, this information allows the bridge to return to the DCPAN and, effectively, allows the communication to be established.

During bridge operation, the selected node hops back and forth between the CPANs, collecting the data in the SCPAN and delivering it to the DCPAN, as shown in the Fig. 1. In general, the superframes of the two CPANs will be skewed in time as the CPANs were formed at different times with different coordinators.

The bridge receives inter-CPAN data from the SCPAN nodes and switches over to the DCPAN for transmission. Since reservation is necessary for any transmission, the bridge switches to the DCPAN and awaits the reservation subframe in order to place a request for transmission, which is performed in the next DCPAN superframe. Upon completing the transmission, the bridge can switch back to the SCPAN for receiving the next batch of inter-CPAN data. However, if the sum of the time lag and the bridge transmission time exceeds one superframe duration, the bridge can't return to the SCPAN in the second superframe (i.e., the one which starts after the bridge has left for reservation). Instead, it will switch back to the third superframe of SCPAN for collecting next batch of inter-CPAN data, as shown in Fig. 1.

Regardless of the amount of inter-CPAN data, the bridge must switch back and forth in order to listen to the trailing and leading beacon in each CPAN: the trailing beacon contains announcements of the next-hop and backup channels, while the leading beacon contains time allocations for transmission (essentially, NAV announcements). 
Bridge transfers are given priority over those of ordinary nodes, as follows. First, the bridge is not required to perform spectrum sensing so it can perform its duties without interruption. Second, the bridge is always allocated transmission slots at the beginning of the data exchange sub-frame, before transmissions by ordinary nodes. This is necessary because of the bridge switching pattern, but sending the data earlier in the superframe means that the probability of a collision with an onset of primary user activity is lower. Finally, the bridge is serviced using exhaustive service policy, which is more efficient than the 1-limited policy applied to the service of ordinary nodes.

\section{ANALYTICAL MODEL OF THE MAC ALGORITHM}

The network environment includes two CPANs with $M$ nodes each; the number includes the coordinator and the bridge which is common to both CPANs. The CPANs need not have the same number of nodes - this would just mean that the bridge will carry different volume of traffic from one CPAN to the other. Time is measured in unit slots and the duration of each superframe is $s_{f}$ slots. Let us assume the time lag between the starting of SCPAN and DCPAN superframes is $\alpha$ basic slots, which is a random value between 0 and the duration of the superframe $s_{f}$. For simplicity, we also assume that data packets have a constant size of $k_{d}$ slots with an immediately following single-slot acknowledgment, for a total of $k_{d}+1$ slots per packet. Let $\lambda$ denote the packet arrival rate per node, assuming Poisson arrivals of data packets, and let $P_{i c}$ denote the fraction of inter-CPAN traffic generated by $M-2$ ordinary nodes in the SCPAN. The arrival rate for the inter-CPAN traffic from SCPAN to DCPAN is, then, $\lambda_{b}=P_{i c}(M-2) \lambda$; the number of nodes of SCPAN greatly impacts the inter-CPAN packet arrival rate of DCPAN.

The timing diagram in Fig. 2 depicts the CPAN service cycle as well as the operation of an arbitrary ordinary node and the bridge node. A CPAN service cycle is defined as the time period between two successive transmissions of the same ordinary node. Due to traffic variability, sensing policy and bridge transmission the CPAN service cycle is a random variable and may take a number of superframes.

A node is allowed to request transmission only for the packets that were in its buffer at the time of the request; packets that arrive to the node during the transmission of earlier requested packets will not be serviced until the next CPAN service cycle. In the same way, the bridge also requests transmission only for the inter-CPAN packets which it has received from the nodes in the SCPAN. Therefore, this scheduling scheme can be modeled as a gated exhaustive policy with vacations [17].

The CPAN coordinator makes a round-robin schedule among the nodes that have been applied in the reservation subframe and announces their transmission slots for the following superframe. The coordinator sorts requests according to roundrobin principle starting with lowest node ID that is larger than the last scheduled ID in previous superframe. Therefore, a target node from SCPAN must wait a random time interval with respect to leading beacon before it starts transmission. However, in the DCPAN, a target node has to wait for an additional time which is due to the bridge transmission. As the bridge has a random number of packets to deliver, this time interval is also random from the standpoint of the target node.

Upon finishing the data transmission the target nodes from both CPANs have to wait for another random time in order to synchronize with beacon. This time interval, which lasts from the end of transmission to the next leading beacon (and, thus, includes the control and reservation sub-frames), is referred to as beacon synchronization.

\section{A. Model of the service period}

The probability generating function (PGF) for the constant packet size with additional acknowledgement is $b(z)=z^{k_{d}+1}$, with the mean value of $\bar{b}=k_{d}+1$. The Laplace-Stieltjes transform (LST) of packet time, $b^{*}(s)=e^{-s\left(k_{d}+1\right)}$, can be obtained by replacing variable $z$ with $e^{-s}$. Therefore, the offered load is $\rho=\lambda \bar{b}$ per node.

The distribution of number of packets that arrive at the node buffer between two successive transmission requests can be represented by the PGF of $\beta(z)=\sum_{k=1}^{\infty} \beta_{k} z^{k}$, where $\beta_{k}$ is the mass probability that $k$ packets are in the buffer when the node applies for bandwidth. Mean number of packets to be transmitted in a single cycle is $\bar{\beta}=\beta^{\prime}(1)=\sum_{k=1}^{\infty} k \beta_{k}$.

The probability distribution of the duration of transmission (service) period can be represented by the PGF $S(z)=$ $\sum_{k=1}^{\infty} s_{k} z^{k}=\beta(b(z))$, as this duration depends on the number of packets arrived during two successive transmission requests and the duration of each packet. Therefore, the LST of the duration of ordinary node service period is $S^{*}(s)=$ $\beta\left(b\left(e^{-s}\right)\right)=\beta\left(b^{*}(s)\right)$ and its mean value is $\bar{S}=\overline{\beta b}$.

\section{B. Duration of bridge transmission}

The time between two successive receptions of inter-CPAN data from SCPAN, hereafter referred to as the bridge cycle, depends on the time lag between CPAN superframes and the duration of bridge transmission. When the two CPANs superframes are not synchronized, i.e., $\alpha>0$, the bridge cycle period lasts two or three superframes depending on $\alpha$ and the bridge transmission time.

- If the sum of $\alpha$ and bridge transmission time is smaller than one superframe duration, the bridge is able to return in the second superframe (which starts after bridge leaves for transmission) of SCPAN for collecting next batch of inter-CPAN data from the third superframe. Therefore, the bridge cycle period lasts two superframes.

- If the sum of $\alpha$ and bridge transmission time is greater than one superframe duration, the bridge will return in the third superframe of SCPAN for collecting next batch of inter-CPAN data from the fourth superframe. This is caused by the time lag, as the second superframe of SCPAN will end before the bridge finishes its transmission in the DCPAN; the bridge will then switch to the SCPAN to listen to the trailing beacon of the second superframe and return to the DCPAN for the remaining transmission. Therefore, the bridge cycle period lasts three superframes. 


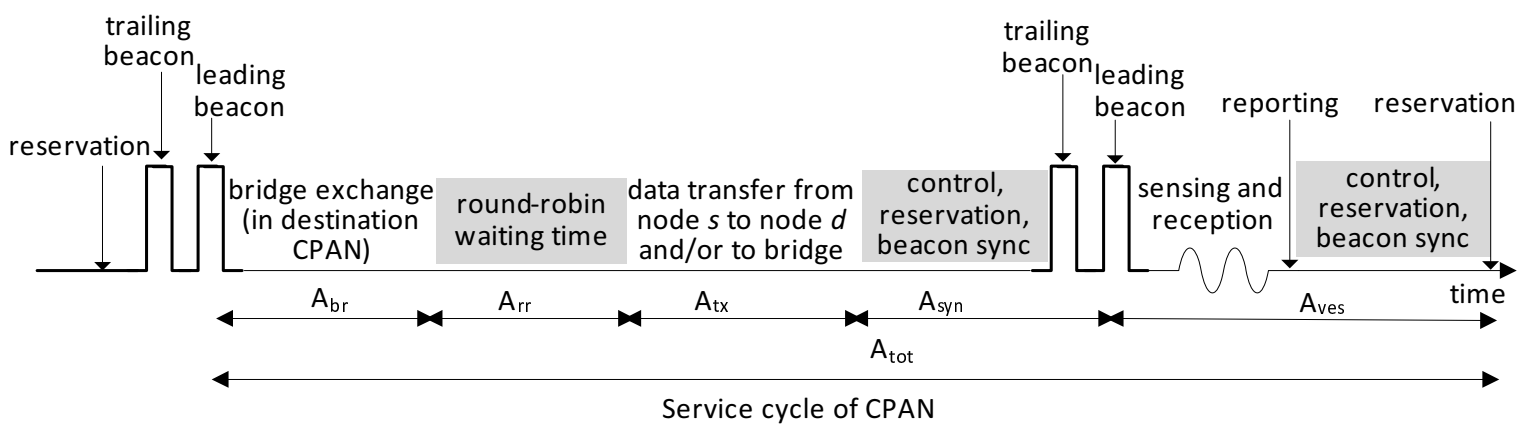

Fig. 2. Packet arrival distribution during service cycle

Let us denote the probability that the sum of $\alpha$ and bridge transmission time is greater than one superframe duration as $P_{b c}$. Then the PGF of bridge cycle period will be

$$
b_{c y c}(z)=P_{b c} z^{3 s_{f}}+\left(1-P_{b c}\right) z^{2 s_{f}}
$$

The Laplace-Stieltjes transform (LST) of bridge cycle period is

$$
b_{c y c}^{*}(s)=b_{c y c}\left(e^{-s}\right)=P_{b c} e^{-s\left(3 s_{f}\right)}+\left(1-P_{b c}\right) e^{-s\left(2 s_{f}\right)}
$$

and its mean value is $\overline{b_{c y c}}=-b_{c y c}^{*^{\prime}}(0)$. The PGF for the number of inter-CPAN packets that arrive to the bridge during this time is

$$
\begin{aligned}
Q b(z) & =b_{c y c}^{*}\left(\lambda_{b}-\lambda_{b} z\right) \\
& =P_{b c} e^{-\left(\lambda_{b}-\lambda_{b} z\right)\left(3 s_{f}\right)}+\left(1-P_{b c}\right) e^{-\left(\lambda_{b}-\lambda_{b} z\right)\left(2 s_{f}\right)}
\end{aligned}
$$

Since each packet needs a service time of $b(z)$ the PGF for the duration of the bridge transmission is

$$
\begin{aligned}
d 2 b(z) & =Q b(b(z)) \\
& =P_{b c} e^{-\left(\lambda_{b}-\lambda_{b} z^{k d}\right)\left(3 s_{f}\right)}+\left(1-P_{b c}\right) e^{-\left(\lambda_{b}-\lambda_{b} z^{k d}\right)\left(2 s_{f}\right)}
\end{aligned}
$$

Therefore, the LST of bridge transmission is

$$
d 2 b^{*}(s)=d 2 b\left(e^{-s}\right)
$$

and its mean value is $\overline{d 2 b}=-d 2 b^{*^{\prime}}(0)$.

However, the distribution of the duration of bridge transmission can also be represented as the series

$$
d 2 b(z)=\sum_{k=0}^{k_{\max }} d_{k} z^{k}
$$

where $d_{k}$ represents the mass probability that bridge transmission takes $k$ slots. The mass probabilities in (6) can be obtained by expanding (4) into the power series in variable $z$. Assuming that the time lag $\alpha$ is uniformly distributed over 0 to $s_{f}-1$, its probability density function (pdf) can be defined as

$$
\alpha(y)=\frac{1}{s_{f}-1}
$$

As the sum of time lag $\alpha$ and duration of bridge transmission $d 2 b$ is a discrete random variable, the mass probability that the sum takes $x$ slots is

$$
h_{x}=\frac{1}{s_{f}-1} \sum_{k=0}^{x} d_{k}
$$

Therefore the probability that the sum of time lag and bridge transmission time is greater than one superframe duration is

$$
\begin{aligned}
P_{b c} & =P\left(X>s_{f}\right)=1-(P(X)<s f) \\
& =1-\sum_{x=0}^{s_{f}-1} h_{x}=1-\frac{1}{s_{f}-1} \sum_{x=0}^{s_{f}-1} \sum_{k=0}^{x} d_{k}
\end{aligned}
$$

The value of $P_{b c}$ can be obtained by substituting the value of $d_{k}$ from (6); in our calculations we assumed that $k_{\max }=2 s_{f}$ (in slots). Therefore, with the known $P_{b c}$ value and by using (5) we can define the PGF for the number of packet arrivals to the ordinary node buffer during the bridge transmission as

$$
A_{b r}(z)=d 2 b^{*}(\lambda-\lambda z) .
$$

However, the bridge transmission will not take place in every superframe of bridge cycle period; it happens in the last superframe of bridge cycle period. Thus the probability that the bridge transmission occurs in the ongoing superframe is $P_{b t}=s_{f} / \overline{b_{c y c}}$. Since the ordinary nodes of DCPAN will not experience bridge transmission delay in every superframe, the PGF for the number of packet arrivals to the ordinary node buffer due to the disruption caused by bridge transmission is

$$
A_{b r_{p}}(z)=P_{b t} A_{b r}(z)+\left(1-P_{b t}\right) .
$$

\section{Round-robin waiting time}

As explained above, round-robin waiting time is the time each ordinary node has to wait while nodes with lower IDs are being serviced. The CPAN service cycle time for two successive transmission opportunities for the ordinary node can be represented by the LST of

$$
C^{*}(s)=\left((1-\rho)+\rho S^{*}(s)\right)^{M-2}
$$

with mean value of $\bar{C}=-C^{*^{\prime}}(0)=\rho(M-2) \bar{S}$. Multiplication with offered load is required because other nodes will transmit only when their respective buffers are not empty. 
In terms of renewal theory [4], elapsed time is the time interval from the beginning of service for nodes with lower IDs (which is considered a renewal point) to an arbitrary point of the CPAN cycle time. The delay observed by a packet, $C_{-}^{*}(s)$, may thus be considered to be the elapsed time of the duration of CPAN cycle time, $C^{*}(s)$, and its LST is

$$
C_{-}^{*}(s)=\frac{1-C^{*}(s)}{s \bar{C}}
$$

with the mean value of $\overline{C_{-}}=\frac{C^{(2)}}{2 \bar{C}}$. The number of packet arrivals during the round-robin service can be described by the PGF of

$$
A_{r r}(z)=C_{-}^{*}(\lambda-\lambda z) .
$$

while the number of packet arrivals during the transmission (service period) can be described by the PGF of $A_{t x}(z)=$ $S^{*}(\lambda-\lambda z)$.

\section{Delay due to beacon synchronization}

In terms of renewal theory [4], residual time is the time interval from an arbitrary moment in a renewal cycle to the beginning of the new renewal cycle. In our scenario, the time from transmitting packet(s) in the current superframe to the next control sub-frame (at which time a node can submit the sensing report to the coordinator) may be considered as residual time, the LST of which is

$$
R_{-}^{*}(s)=\frac{1-e^{-s s_{f}}}{s s_{f}} .
$$

Hence, the PGF for the number of packet arrivals during this time is $A_{\text {syn }}(z)=R_{-}^{*}(\lambda-\lambda z)$.

\section{E. Duration of sensing}

The coordinator assigns sensing duty to the nodes that have transmitted their packets, and the duration of this duty, expressed in superframes, is the product of sensing penalty $k_{p}$ and the number of packets $k$ transmitted in the service period. Thus, the distribution of time spent in sensing can be represented by the PGF of $V(z)=\sum_{k=1}^{\infty} \beta_{k} z^{k_{p} s_{f} k}=\beta\left(z^{k_{p} s_{f}}\right)$ and its mean value is $\bar{V}=k_{p} s_{f} \bar{\beta}$. The corresponding LST of a single sensing period is $V^{*}(s)=\sum_{k=1}^{\infty} \beta_{k} e^{-k_{p} s_{f} k}$. The number of packets that arrive during the sensing period can be obtained by replacing $s$ with $\lambda-\lambda z$ in the last equation, i.e.,

$$
A_{v c}(z)=V^{*}(\lambda-\lambda z)=\sum_{k=0}^{\infty} f_{k} z^{k}
$$

where $f_{k}$ represents the mass probability of $k$ packets arrivals during the sensing period.

\section{F. Impact of packet reception during sensing}

A node can suspend an ongoing sensing in order to receive packet(s). Packet reception may take place in one or more superframes. However, the node has to finish its sensing duty before placing a new transmission request. As the result, reception will effectively extend the duration of the sensing period. To model this effect, we need to find the probability of packet reception during the sensing period.

Each of the nodes in the SCPAN generates intra-CPAN traffic at a rate of $\frac{\lambda\left(1-P_{i c}\right)}{M-2}$, assuming uniform distribution of destinations in each CPAN. Probability of having no packets for a given target node during a sensing period is $P_{n r s}=e^{-\frac{\lambda\left(1-P_{i c}\right)}{M-2} \bar{V}}$, and the PGF for extended sensing period due to reception is $V_{e s}(z)=P_{n r s}+\left(1-P_{n r s}\right) z^{s_{f}}$.

However, a node in the DCPAN receives intra-CPAN traffic at a rate of $\frac{\lambda}{M-2}$, and inter-CPAN packets at a rate of $\frac{\lambda_{b}}{M-2}$. Probability of having no packets during the sensing period is $P_{n r d}=e^{-\frac{\lambda_{b}}{M-2} \overline{b_{c y c}}} e^{-\frac{\lambda}{M-2} \bar{V}}$. Therefore, the PGF for extended sensing period in the DCPAN due to reception is $V_{e s}(z)=$ $P_{n r d}+\left(1-P_{n r d}\right) z^{s_{f}}$. The number of packets that arrive during this extended sensing period can be described by the PGF of $A_{\text {ves }}(z)=V_{\text {es }}(\lambda-\lambda z)$.

\section{G. Time between successive transmission requests}

The PGF for the number of packet arrivals to a node during the interval between two successive bandwidth requests is

$$
Q(z)=A_{b r_{p}}(z) A_{r r}(z) A_{t x}(z) A_{s y n}(z) A_{v e s}(z)=\sum_{k=0}^{\infty} q_{k} z^{k} .
$$

If the node finishes sensing and has no packets in its buffer, it will continue with sensing duty, which occurs with the probability of $q_{0}=Q(0)$. The distribution of the number of packets that arrive at the ordinary node buffer between two successive transmission requests can be described by the PGF of

$$
\beta(z)=\frac{Q(z)-q_{0}}{1-q_{0}}
$$

and its mean value is $\bar{\beta}=\bar{A}_{b r_{p}}+\bar{A}_{r r}+\bar{A}_{t x}+\bar{A}_{s y n}+\bar{A}_{v e s}$.

The PGF for the total sensing period is

$$
V_{t o t}(z)=\frac{V_{e s}\left(1-q_{0}\right)}{1-V_{e s} q_{0}} .
$$

The equations presented here can be solved as a system with unknowns $\beta_{k}, k=1 \ldots n_{c}$, if we limit the number of terms in each PGF or LST to $n_{c}$.

\section{H. Probability of incomplete transmission}

Transmissions may be interrupted by primary user activity in two ways. First, the CPAN may hop onto a channel which is already used by a primary source; this may be due to an error in the channel table (i.e., a channel is recorded as idle whereas it is actually busy) or the onset of primary user activity after the channel has been sensed. However, the channel which was idle at the beginning of a superframe may become busy during an ongoing data transmission. To model the probability of these two mechanisms that lead to incomplete transmission, we have to consider the following.

The CPANs operate on $N$ RF channels, each with a separate primary source which exhibit random ON and OFF periods. The pdf of ON and OFF periods are $g_{o n}(x)$ and $g_{o f f}(x)$, and the corresponding mean values are $\bar{G}_{o n}$ and $\bar{G}_{o f f}$, respectively. Therefore the mean cycle time of primary source 
activity is $\bar{G}_{c y l}=\bar{G}_{o n}+\bar{G}_{o f f}$, while its activity probability (duty cycle) is $p_{o n}=\frac{\bar{G}_{o n}}{\bar{G}_{c y c}}$.

The CPAN superframe starts from a random point in the idle (OFF) channel period and continues until it finishes or the channel becomes active (ON). In the latter case, the superframe collides with the primary source and we need to find the residual channel idle time. The pdf of residual channel idle time is proportional to the probability that channel idle time is larger than some value $y$. This pdf can be represented as $f(y)=\frac{\int_{z=y}^{\infty} g_{o f f}(z) d z}{\bar{G}_{\text {off }}}$. Therefore the probability distribution function (PDF) of residual channel idle time is $F(x)=\int_{0}^{x} d(y) d y$.

1) Probability that coordinator has inaccurate channel information: Probability of ordinary node performing channel sensing is

$$
P_{s}=\frac{\bar{V}_{t o t}}{\overline{d 2 b}+\bar{C}_{-}+\bar{S}_{-}+\bar{R}_{-}+\bar{V}_{t o t}}
$$

Probability distribution of the number of nodes concurrently performing channel sensing is described with the PGF of

$$
\Theta(z)=\sum_{i=0}^{M-1}\left(\begin{array}{c}
M-1 \\
i
\end{array}\right) P_{s}^{i}\left(1-P_{s}\right)^{M-1-i} z^{i}=\sum_{n=0}^{M-1} \theta_{n} z^{n}
$$

where $\theta_{n}$ represents the mass probability that $n$ nodes are performing channel sensing.

To find the probability distribution of the time between consecutive sensing events we will assume that the node chooses randomly the channel to sense among all channels except the one currently used by the CPAN, so that the probability of sensing any given channel is $P_{j}=\frac{1}{N-1}$. Sensing one channel is assumed to last for $d$ slots.

Then, using (21) and the assumptions above, the PGF of the time period of two consecutive sensing events on a particular channel is

$$
\begin{aligned}
\Omega(z) & =\theta_{0} \sum_{k=1}^{\infty} P_{j}\left(1-P_{j}\right)^{k-1} z^{k} d \\
& +\sum_{l=1}^{\min (M-1, N-2)} \theta_{l} \sum_{k=1}^{\infty} l P_{j}\left(1-l P_{j}\right)^{k-1} z^{k} d \\
& +\sum_{\min (M-1, N-2)+1}^{M-1} z^{d}
\end{aligned}
$$

where typically $M<N-1$. By applying renewal theory and the steps derived in [9]. we can calculate the probability $P_{i}$ of having inaccurate channel status in the channel table (due to the channel becoming active between two consecutive sensing events) and its duration in the channel table.

2) Probability of collision during transmission: The primary source can become active in any moment, hence we need to find the probability that channel will become active during an ongoing data transmission. As the CPAN superframe starts at a random point in the idle channel period, the collision occurs if the residual channel idle time is shorter than the superframe duration. Therefore the collision probability of bridge transmission and ordinary node transmission can be deduced as follows:

$$
\begin{gathered}
P_{c}^{(b r)}=\int_{x=0}^{\infty}(D(x+\overline{d 2 b})-D(x)) d(x) d x \\
P_{c}^{(o)}=\int_{x=0}^{\infty}\left(D\left(x+\overline{d 2 b}+\bar{C}_{-}+\bar{S}\right)-D(x)\right) d(x) d x
\end{gathered}
$$

As the bridge transmits immediately after the beacon and before the transmission of ordinary nodes, it will suffer fewer collisions with the primary source activity than ordinary nodes. We note that this type of collision will make the channel unusable and recovery procedure will be necessary [11].

Total collision probability for bridge node as well as other ordinary nodes is, then, the sum of the probability of inaccurate channel information and probability of collision during transmission:

$$
\begin{gathered}
P_{c o l}^{(b r)}=P_{c}^{(b r)}+P_{i} \\
P_{c o l}^{(o)}=P_{c}^{(o)}+P_{i}
\end{gathered}
$$

\section{PACKET ACCESS DELAY}

\section{A. Intra-CPAN packet delay}

Let us assume that an ordinary node in the SCPAN has already $L^{*}$ intra-CPAN packets at the moment it applies for bandwidth. Let us also assume that $A_{i}$ packets arrive to the node while it is transmitting the $i^{\text {th }}$ packet. Thus after transmitting the $n^{\text {th }}$ packet in the transmission (service) period the buffer has $L_{n}=L^{*}+A_{b r_{p}}+A_{r r}+A_{1}+A_{2}+\ldots+A_{n}-n$ packets, and the PGF of the number of packets left after $n^{\text {th }}$ departing packet can be obtained as

$$
L_{n}(z)=\frac{A_{b r_{p}}(z) A_{r r}(z) A(z)^{n} \sum_{k=n}^{\infty} \beta_{k} z^{k}}{z^{n} \sum_{k=n}^{\infty} \beta_{k}}
$$

From this equation and the single packet serving time $b^{*}(\lambda-$ $\lambda z)$, we can obtain the PGF of the number of packets left in the buffer after any departing packet as

$$
\begin{aligned}
L(z) & =\sum_{n=1}^{\infty} \frac{\sum_{k=n}^{\infty} \beta_{k}}{\bar{\beta}} L_{n}(z) \\
& =A_{b r_{p}}(z) A_{r r}(z) \frac{\left(\beta\left[b^{*}(\lambda-\lambda z)\right]-\beta(z)\right) b^{*}(\lambda-\lambda z)}{\bar{\beta}\left[b^{*}(\lambda-\lambda z)-z\right]}
\end{aligned}
$$

Packets are serviced in FIFO order and the number of packets left after a departing packet is equal to the number of packets that arrived during the departing packet was in the system. The probability distribution of packet waiting time can be obtained from

$$
L(z)=T_{a}^{*}(\lambda-\lambda z)=W^{*}(\lambda-\lambda z) b^{*}(\lambda-\lambda z)
$$

and the corresponding LST of intra-CPAN packet waiting time is

$$
W^{*}(s)=A_{b r_{p}}\left(1-\frac{s}{\lambda}\right) A_{r r}\left(1-\frac{s}{\lambda}\right) \frac{\lambda\left(\beta\left[b^{*}(s)\right]-\beta\left(1-\frac{s}{\lambda}\right)\right)}{\bar{\beta}\left[\lambda b^{*}(s)-\lambda+s\right]}
$$

with the mean value of $\bar{W}=\left.\frac{d W^{*}}{d s}\right|_{s=0}=\frac{(1+\rho) \beta^{(2)}(1)}{2 \lambda \bar{\beta}}$. 


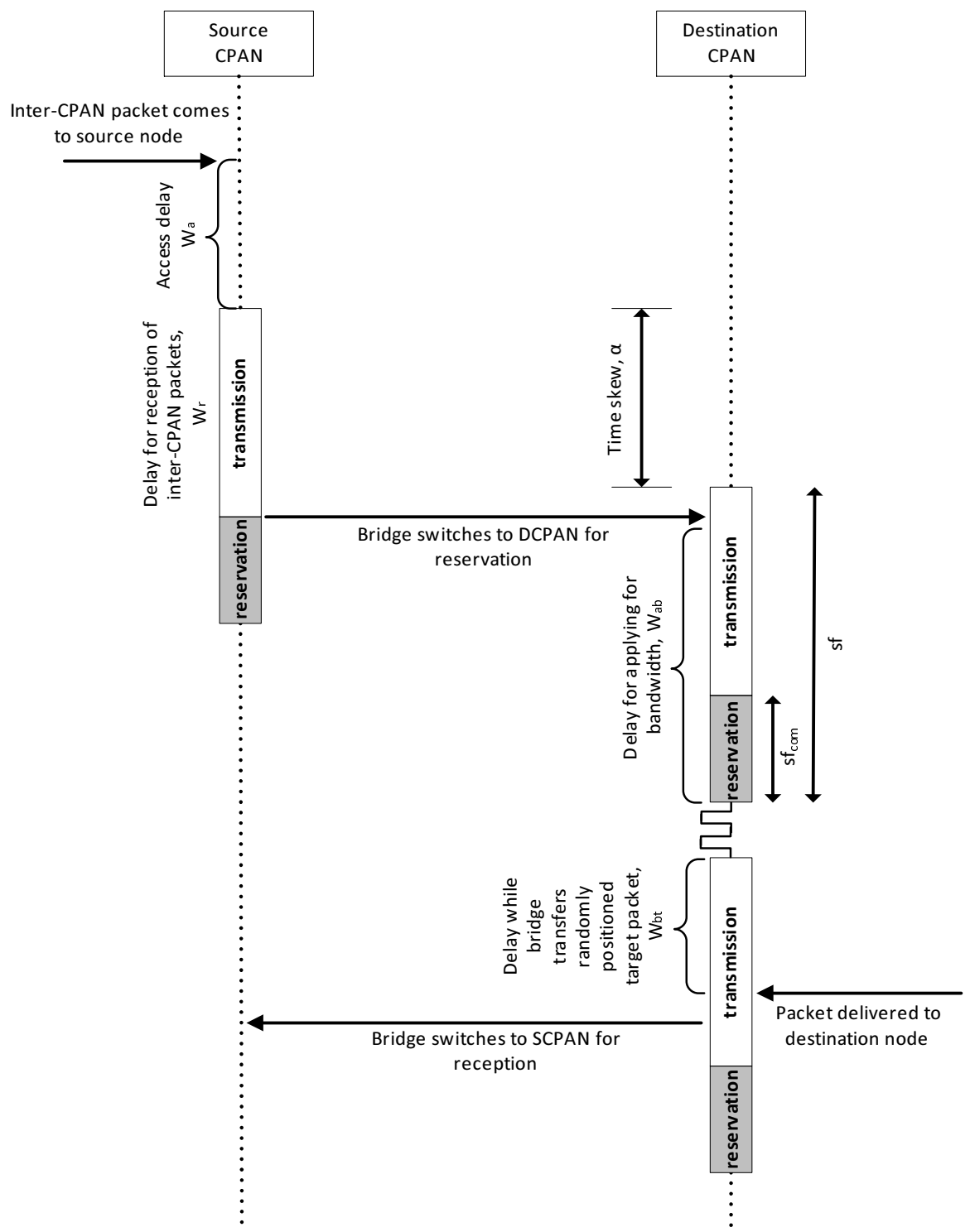

Fig. 3. End-to-End delay for Inter-CPAN packet

\section{B. Inter-CPAN packet delay}

Before calculating the inter-CPAN packet delay, we note that an inter-CPAN packet, from its arrival to the source node to the reception by the destination node, undergoes four different phases as shown in Fig. 3.

First the packet has to wait to be transmitted to the bridge. This time, commonly referred to as access delay $\left(W_{a}\right)$, is equal to the residual time of bridge cycle period $W_{a}^{*}(s)=\frac{1-b_{c y c}^{*}(s)}{s \bar{b}_{c y c}}$, with mean value of $\bar{W}_{a}=-W_{a}^{*^{\prime}}(0)$.

Second, it waits until the bridge finishes receiving all of the inter-CPAN packets from the SCPAN, which lasts for one entire transmission sub-frame: $W_{r}=s_{f}-s_{\text {com }}$.

Third, the bridge switches to DCPAN to apply for bandwidth. Due to time lag $\alpha$ between the beacons of two CPANs, the time delay to the actual application for bandwidth varies: if $\alpha<W_{r}$, the delay will be $s_{f}-\left(W_{r}-\alpha\right)$, otherwise the delay will be $\left(\alpha-W_{r}\right)+s_{f}$. The probability of $\alpha$ being lower than $W_{r}$ is

$$
\begin{aligned}
P_{a b} & =P\left(\alpha<W_{r}\right)=\int_{u=0}^{W_{r}} P(\alpha<u) P\left(W_{r}=u\right) d u \\
& =\int_{u=0}^{W_{r}} \frac{d u}{s f}=\frac{W_{r}}{s_{f}}
\end{aligned}
$$

Thus, the PGF for this waiting time is $W_{a b}(z)=$ $P_{a b} z^{s_{f}-\left(W_{r}-\alpha\right)}+\left(1-P_{a b}\right) z^{\left(\alpha-W_{r}\right)+s_{f}}$ and its mean value is $\bar{W}_{a b}=W_{a b}^{\prime}(1)$.

Finally, the bridge has to deliver its packets in the DCPAN. Due to round-robin service policy, packets are randomly positioned within the bridge queue. The delay experienced by a bridge packet in the DCPAN, $W_{b t}$, is $W_{b t}^{*}(s)=\frac{1-d 2 b^{*}(s)}{s \overline{d 2 b}}$ (where $d 2 b^{*}(s)$ denotes the elapsed time of the duration of bridge transmission), and its mean is $\bar{W}_{b t}=-W_{b t}^{*^{\prime}}(0)$.

Therefore, mean end-to-end delay for an inter-CPAN packet can be obtained as $\bar{W}_{a}+W_{r}+\bar{W}_{a b}+\bar{W}_{b t}$. 


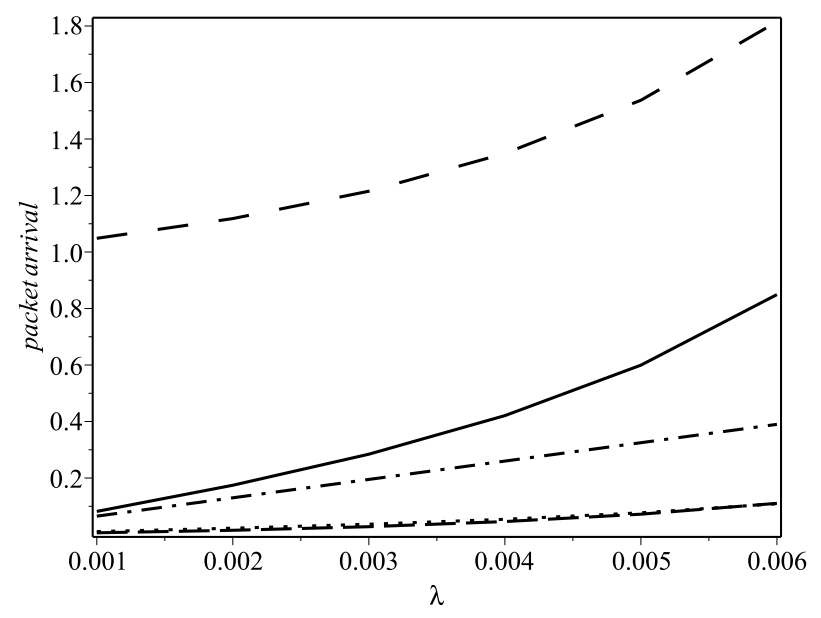

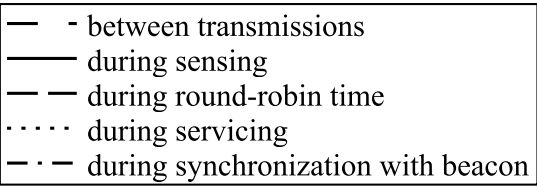

Fig. 4. Average number of packet arrivals during different intervals in the CPAN.

\section{PERFormance AnAlysis}

To assess the performance of the proposed bridging scheme, we have solved the system of equations described above using Maple 16 from Maplesoft, Inc. [8]. We have assumed that the network uses $N=19$ channels. However, the number of channels can vary depending on the operational range of frequency band $(54-862 \mathrm{MHz})$ and the bandwidth $(5-8 \mathrm{MHz})$ of each channel [2]. Each of the channel is intermittently (and randomly) occupied by a dedicated primary source. Mean cycle time of primary source has been set to $G_{c y l}=3000$ time units where $\mathrm{ON}$ and OFF periods are exponentially distributed with mean values of 900 and 2100 time units, respectively. By considering a capacity of about $19.8 \mathrm{Mbps}$ per $6 \mathrm{MHz}$ channel, we have assumed the packet size is 1 Kbyte and it needs $k_{d}=10$ time units to be transmitted. The size of the superframe is 130 time units, 30 of which are allocated for the beacon, control and reservation sub-frames. The time lag between CPAN superframes is $s_{f} / 2=65$ time units. The number of nodes for each CPAN is $M=14$, while the sensing penalty is set to $k_{p}=0.6$.

\section{A. Performance without the bridge}

Our first experiment tries to establish a baseline by considering the performance of the CPAN without bridge transmissions.

Fig. 4 shows the average number of packet arrivals to an ordinary node in the CPAN during different stages of node activities. As expected, the average number of packet increases when traffic intensity $(\lambda)$ increases. However, the probability of inter-CPAN traffic $P_{i c}$ doesn't impact the arrival of packets to the node buffer as bridge node only receives inter-CPAN packets from SCPAN.

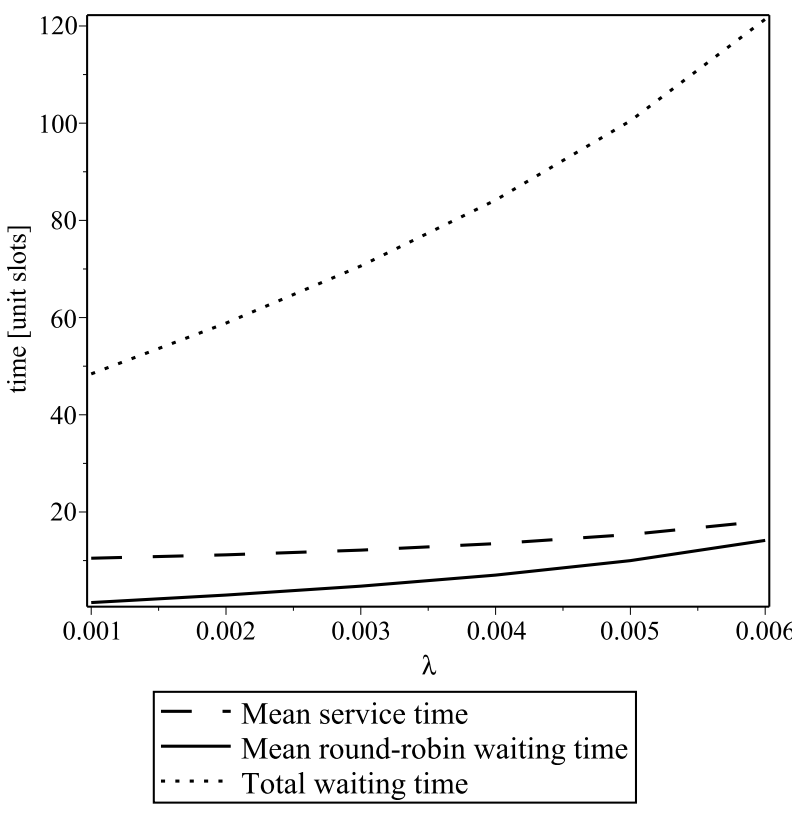

Fig. 5. Packet delays for ordinary node transmission in the SCPAN.

Fig. 5 shows the average duration of service period, cycle period and waiting time for ordinary nodes transmission in the source CPAN. Servicing a higher number of packets makes the node experiences longer service duration and longer roundrobin waiting time (i.e., waiting for the nodes with lower IDs) which eventually increases the mean packet waiting time.

These results hold for both SCPAN and DCPAN in the absence of bridge transmissions.

\section{B. The impact of bridge activity}

However, the performance indicators will change when the bridge is active. In our second experiment we have varied the packet arrival rate and the probability of inter-CPAN traffic, and examined the behavior of the DCPAN.

Fig. 6 shows the average number of packet arrivals to the node buffer during different stages of node activities in the DCPAN. As expected, the average number of packet increases with traffic intensity $\lambda$ and the probability of inter-CPAN traffic $P_{i c}$. Of course, higher values of $P_{i c}$ lead to longer bridge exchanges, which explains the shape of the surface in Fig. 6(a). The increase in Fig. 6(b) is due to a more subtle mechanism: namely, at higher values of $\lambda$, an increase in $P_{i c}$ means that the bridge needs more transmission time in the DCPAN superframe, which leaves less time for local DCPAN traffic due to higher priority of bridge transmissions. Then, time between two successive transmission opportunities for ordinary nodes in the DCPAN gets longer, and mean packet arrival rate tends to increase.

Fig. 7 shows the duration of the bridge exchange as well as the performance indicators for ordinary node transmissions in the DCPAN. Since bridge transmissions are given priority, the performance of ordinary nodes is affected by the bridge transmission. More packet arrivals lead to longer bridge exchanges, Fig. 7(a); as less time is left for ordinary nodes, higher traffic 


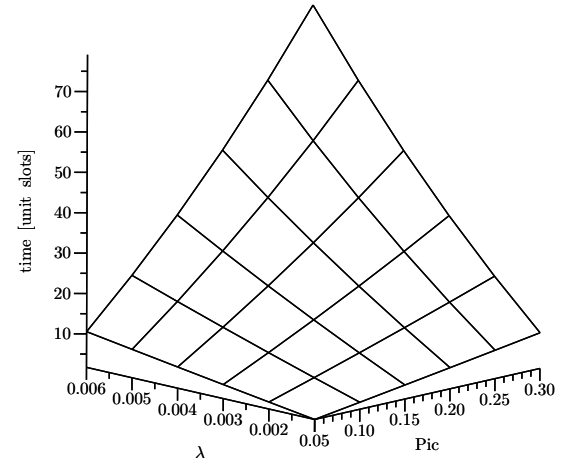

(a) Mean duration of bridge exchange.

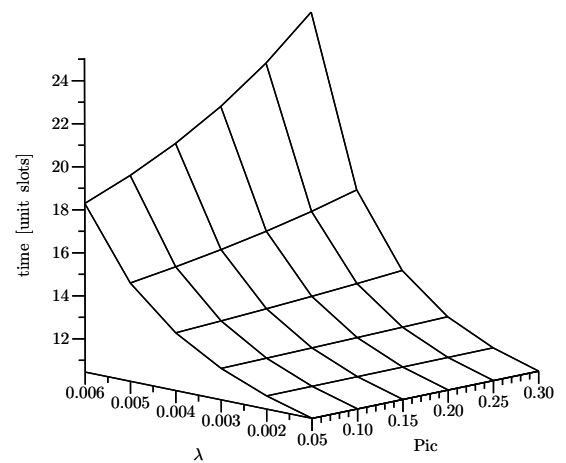

(b) Mean transmission time.

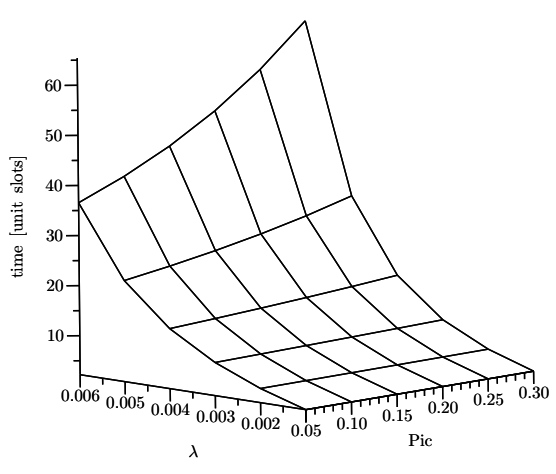

(c) Mean cycle time.

Fig. 7. Performance of data transmission in the destination CPAN.

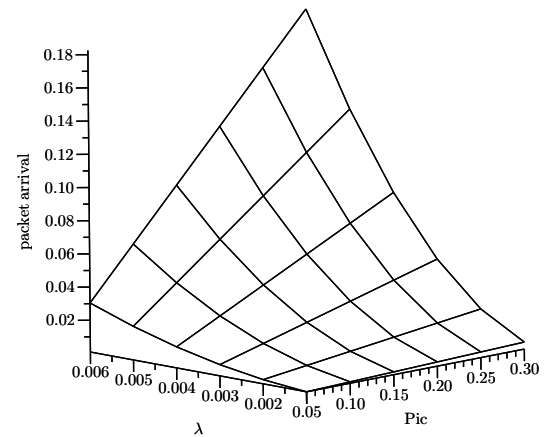

(a) During bridge exchange.

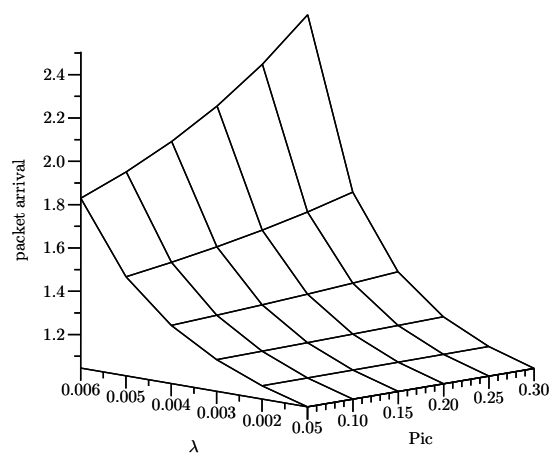

(b) Between two successive transmission requests.

Fig. 6. Mean number of packet arrivals in the DCPAN.

load leads to longer transmission and cycle times. This effect is somewhat moderated by the decrease in local traffic caused by higher value of $P_{i c}$, as can be seen in Figs. 7(b) and 7(c), respectively.

Fig. 8 shows mean bridge cycle period and mean delay for

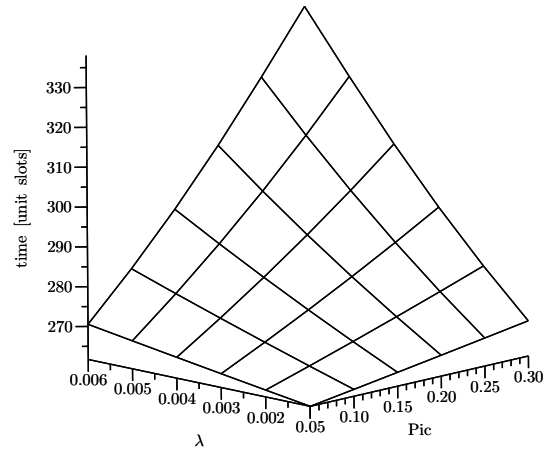

(a) Mean bridge cycle period.

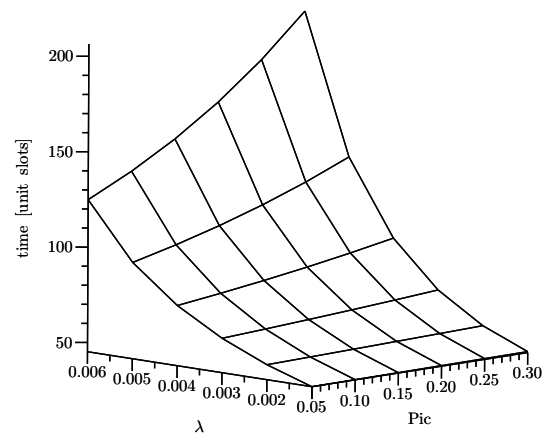

(b) Mean duration of intra-CPAN packet waiting time.

Fig. 8. Average bridge cycle period and intra-CPAN packet waiting time in the DCPAN.

intra-CPAN traffic. Obviously the bridge cycle period strongly depends on traffic intensity and probability of inter-CPAN traffic, as does the mean delay for intra-CPAN traffic which is further affected by the round-robin waiting time. Note that, 


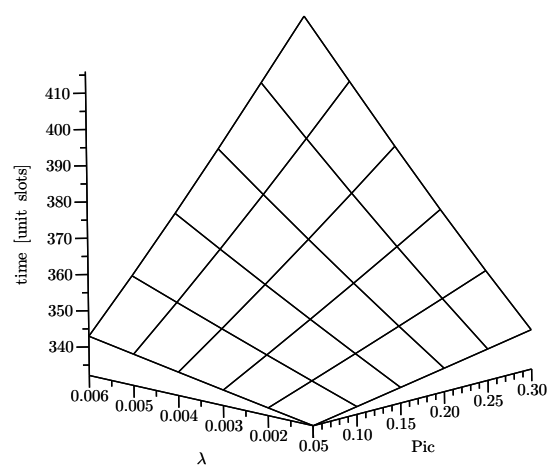

(a) As function of packet arrival rate and probability of inter-CPAN traffic, with time lag fixed at $\alpha=$ $s_{f} / 2$.

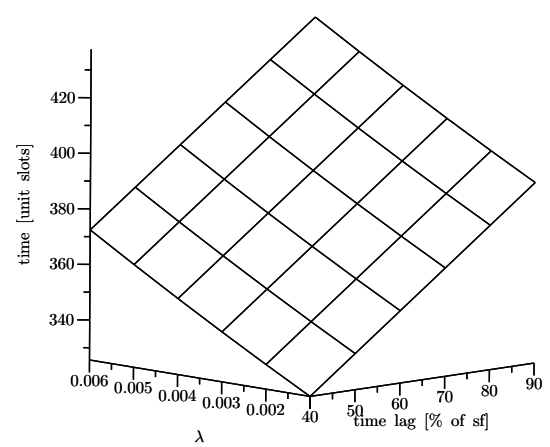

(b) As function of packet arrival rate and time lag, with probability of inter-CPAN traffic fixed at $P_{i c}=$ 0.2 .

Fig. 9. Mean end-to-end delay for inter-CPAN traffic.

as the bridge transmission time increases due to the increase of inter-CPAN traffic, the bridge cycle period increases and eventually exceeds three superframe durations. Fig. 8(b) shows the mean intra-CPAN packet waiting time which is affected by the bridge transmission and round-robin waiting time.

Fig. 9 shows mean end-to-end delay for inter-CPAN traffic. In case the time lag between two superframes is fixed, as shown in Fig. 9(a), the delay increases with both packet arrival rate and probability of inter-CPAN traffic, since an inter-CPAN packet would have to wait longer in the bridge queue when $\lambda$, $M$, and/or $P_{i c}$ increase. In case the probability of inter-CPAN traffic is fixed while the time lag is variable, Fig. 3, values of time lag that deviate from the value of $s_{f} / 2$ mean that there is less time in one or the other CPAN for bridge transmissions, which may easily led to the extension of the bridge cycle and longer end-to-end delays for inter-CPAN traffic.

We note that the delay for inter-CPAN traffic is much higher than that of intra-CPAN traffic. This is not unexpected since the bridging protocol requires that the bridge hops from one CPAN to another, and it has to follow the superframe structure in both of them. However, the rate of change of the delay vs.

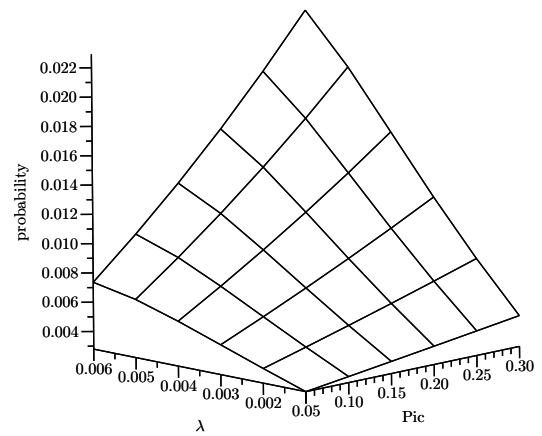

(a) probability of bridge collision

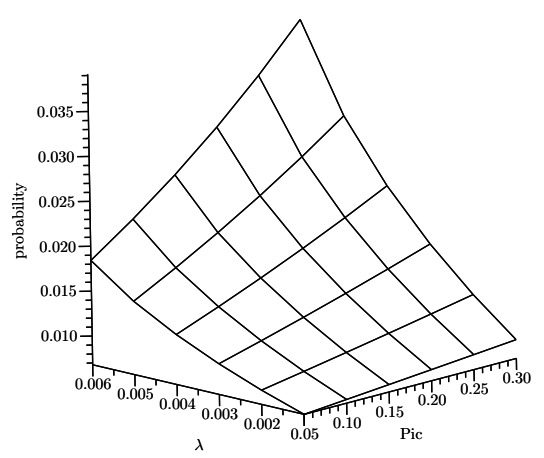

(b) probability of ordinary node collision

Fig. 11. Collision probability with primary source

both traffic intensity and probability of inter-CPAN traffic is much lower for the inter-CPAN traffic, Fig. 9(b), than for the intra-CPAN one, Fig. 8(b), which provides further proof of the efficiency of the proposed protocol.

\section{Impact of collisions with primary source}

We have also investigated the impact of collisions with primary source activity for both bridge and ordinary node transmissions.

Fig. 10 shows the average number of nodes that perform sensing, average interval between consecutive sensing events and the probability of inaccurate information in the channel table. Under low loads, most of the nodes perform sensing and most channels are sensed rather frequently; as the result, the probability of inaccuracies in the channel table is low. As traffic intensity increases, nodes have more data to transmit and, consequently, spend less time in sensing. This effect is somewhat countered by bridge activity: namely, higher interCPAN traffic leaves fewer opportunities for ordinary nodes to request bandwidth and they perform more sensing. Overall, the number of sensing nodes exhibits a slight increase with higher inter-CPAN traffic, at very high traffic load. This increase translates into flattening and even a slight decrease of the mean interval between sensing events and probability of inaccuracies in the channel table. 


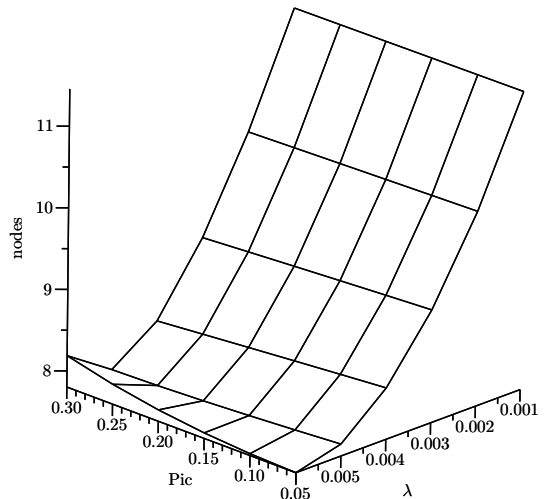

(a) Mean number of nodes performing sensing.

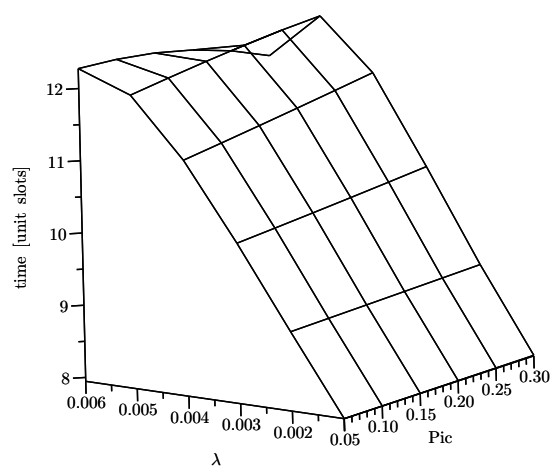

(b) Mean time between consecutive sensing events.

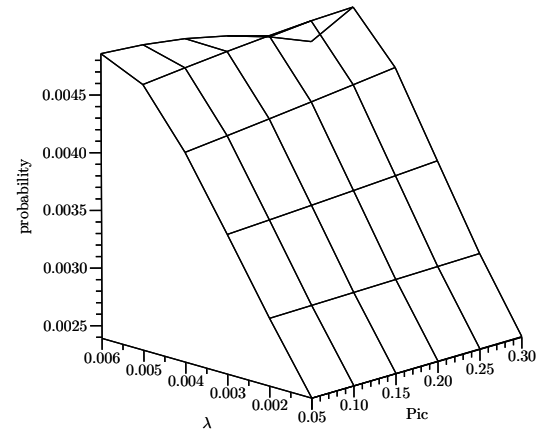

(c) Probability of inaccurate channel table.

Fig. 10. Performance of the sensing process.

The resulting collision probability is shown in Fig. 11. As bridge transmissions take place before those of ordinary nodes, the collision probability of bridge transmissions is somewhat smaller; nevertheless, both probabilities increase with traffic intensity and probability of inter-CPAN traffic.

\section{CONCLUSION AND FUtURE WORK}

In this paper, we have described and analyzed the performance of a bridging mechanism for two cognitive piconets with non-synchronized superframes. We have shown that the performance for both intra- and inter-CPAN traffic is dependent on traffic and network parameters, in particular traffic intensity, probability of non-local traffic, and time lag between CPAN superframes.

Our future work will focus on finding optimum network parameters that would minimize the end-to-end delay for interCPAN packets. We also plan to work on reliable mechanisms for CPAN discovery and synchronization, and to apply these findings in the design of a holistic routing protocol for cognitive networks.

\section{REFERENCES}

[1] I. F. Akyildiz, W.-Y. Lee, M. C. Vuran, and S. Mohanty. NeXt generation/dynamic spectrum access/cognitive radio wireless networks: A survey. Computer Networks, 50:2127-2159, 2006.

[2] C. Cordeiro, K. Challapali, and M. Ghosh. Cognitive PHY and MAC layers for dynamic spectrum access and sharing of TV bands. In Proceedings of the first international workshop on Technology and policy for accessing spectrum, page 3. ACM, 2006.

[3] I. Filippini, E. Ekici, and M. Cesana. A new outlook on routing in cognitive radio networks: Minimum-maintenance-cost routing. ACM/IEEE Transactions on Networking, 21(5):1484-1498, 2013.

[4] D. P. Heyman and M. J. Sobel. Stochastic Models in Operations Research, Volume I: Stochastic Processes and Operating Characteristics. McGraw-Hill, New York, 1982.

[5] X. Huang, D. Lu, P. Li, and Y. Fang. Coolest path: spectrum mobility aware routing metrics in cognitive ad hoc networks. In 31st Int. Conf. Distributed Computing Systems (ICDCS), pages 182-191, Minneapolis, MN, 2011.

[6] P. K. Lee. Joint frequency hopping and adaptive spectrum exploitation. In IEEE Military Communications Conference MILCOM2001, volume 1, pages 566-570, Washington, DC, Oct. 2001.
[7] Y. Liu, L. X. Cai, and X. Shen. Spectrum-aware opportunistic routing in multi-hop cognitive radio networks. Selected Areas in Communications, IEEE Journal on, 30(10):1958-1968, 2012.

[8] Maplesoft, Inc. Maple 16. Waterloo, ON, Canada, 2013.

[9] J. Mišić and V. B. Mišić. Performance of cooperative sensing at the MAC level: Error minimization through differential sensing. IEEE Transactions on Vehicular Technology, 58(5):2457-2470, June 2009.

[10] J. Mišić and V. B. Mišić. Simple and efficient MAC for cognitive wireless personal area networks. In Proc. Global Telecommunications Conference GLOBECOM'09, Honolulu, HI, Nov. 2009.

[11] J. Mišić and V. B. Mišić. Recovery in channel-hopping cognitive networks under random primary user activity. IEEE Transactions on Vehicular Technology, 63(5):2392-2406, June 2014.

[12] J. Mišić, V. B. Mišić, and M. S. I. Khan. On the selection of working channels in a channel-hopping cognitive PAN. In 9th Int. Wireless Communications and Mobile Computing Conf. (IWCMC 2013), Cagliari, Sardinia, Italy, July 2013.

[13] M. Pan, P. Li, Y. Song, Y. Fang, and P. Lin. Spectrum clouds: A session based spectrum trading system for multi-hop cognitive radio networks. In INFOCOM, 2012 Proceedings IEEE, pages 1557-1565. IEEE, 2012.

[14] M. Pan, C. Zhang, P. Li, and Y. Fang. Joint routing and link scheduling for cognitive radio networks under uncertain spectrum supply. In Proc. INFOCOM, pages 2237-2245, Shanghai, China, 2011.

[15] M. M. Rahman, J. Mišić, and V. B. Mišić. Performance of bridging in cognitive wireless personal area networks. In IEEE Conference on Wireless Communications and Networking Conference (WCNC 2015), New Orleans, LA, The USA, Mar. 2015.

[16] Y. Shi and Y. T. Hou. A distributed optimization algorithm for multi-hop cognitive radio networks. In Proc. INFOCOM, Phoenix, AZ, 2008.

[17] H. Takagi. Queueing Analysis, volume 1: Vacation and Priority Systems. North-Holland, Amsterdam, The Netherlands, 1991. 